\title{
COMBINED EFFECT OF THERMAL RADIATION AND VISCOUS DISSIPATION ON UNSTEADY FREE CONVECTIVE MAGNETO- HYDRODYNAMIC FLOW THROUGH AN IRREGULAR CHANNEL
}

\author{
T. L. Oyekunle, S. A. Agunbiade* \\ ${ }^{1}$ Department of Mathematics, Faculty of Physical Sciences, University of Ilorin, P.M.B. 1515, \\ Ilorin, Nigeria. \\ e-mail: tloyekunle95@gmail.com; agunbiade1971@gmail.com \\ *corresponding author
}

\begin{abstract}
This study is focused on the joint effects of thermal radiation and magnetic field on an unsteady flow of natural convective, electrically conducting fluid past an irregular vertical channel in the presence of viscous dissipation. The magnetic field is applied normal to the channel. The coupled ordinary differential equations (ODE) are obtained using perturbation technique on the dimensionless governing equations involving energy and momentum equation. Semi-analytical solutions are obtained for the resultant coupled ODE with the application of Adomian Decomposition Method (ADM) with the aid of Mathematica 11.0 software. The pertinent flow parameters in velocity, temperature, Skin-friction and Nusselt number are discussed and exhibited using tables and graphs. It is revealed that at all regions, thermal radiation and viscous dissipation have a tendency to accelerate the Skin-friction and heat transfer coefficient of the fluid.
\end{abstract}

Keywords: Thermal radiation, Viscous dissipation, Adomian decomposition method (ADM), Constant Magnetic field and Irregular channel.

\section{Introduction}

In the fluid flow and heat transfer process, thermal radiation plays a prominent role. In designing of advanced energy conversion systems that operate at high temperatures, the effect of thermal radiation can not be over-emphasized. It has its applications in areas such as: engineering, aerodynamics, missiles, nuclear physics. Sivaiah and Reddy (2017) investigated MHD unsteady radiating fluid flow through an inclined permeable porous plate in the presence of Hall current. They reported that velocity is enhanced with increase in radiation parameter, while temperature profiles are slowed down. Dada and Agunbiade (2016) examined Rivlin-Ericksen fluid with the impacts of thermal radiation and chemical reaction on free convective flow through a vertical permeable plate. Effects of radiation on forced convective MHD flow in a Darcy-forchheimer permeable medium with heat transfer was discussed by Jhankal et al. (2017). Runge-Kutta fourth order method was used to obtain the solution to the problem. The result revealed that radiation 
influenced the flow significantly. Zigta and Koya (2017) considered the effects of chemical reaction and thermal radiation on free convective MHD with periodic temperature. The results showed that a hike in Grashof number accelerated the velocity profiles. Hall current effect in the three-dimensional squeezing rotating mixed convection flow in the presence of heat sources and thermal radiation was studied by Suguna and Bhuvana (2019). Rao (2018) examined chemical reaction and thermal radiation effects on MHD Casson fluid flow through an inclined exponentially stretching surface. Raju (2018) presented effects of radiation and chemical reaction on the mass and heat transfer MHD convective flow through a porous vertically semi-infinite moving plate. It was observed that the increase in radiation accelerated velocity and temperature profiles.

Moreover, the combined effect of viscous dissipation and thermal radiation on the fluid flow are highly significant as a result of its applications in moderating the cooling rate. Mostafa et al. (2017) analysed the joint effect of radiation and viscous dissipation on free convective unsteady Non-Newtonian fluid through a vertically continuous moving stretched surface in the presence of No-Slip phenomena. The study concluded that temperature distributions were slowed down in the presence of radiation parameter. Viscous dissipation effects on convective rotatory RivlinEricksen flow via a permeable vertical plate with chemical reaction was investigated by Agunbiade and Dada (2019). It was reported that increase in viscous dissipation accelerated resultant velocity of the fluid. Nagasasikala and Lavanya (2018) discussed radiation and dissipation effects on heat transfer flow in a rotating convective cuo-water Nano-fluid past a vertical channel. These results agreed with Mostafa et al. (2017) that temperature profiles are retarded by radiation parameter. Kumar and Reddy (2018) considered combined effects of thermal radiation and viscous dissipation on convective MHD flow through a vertical permeable plate. Emeka (2019) studied the effect of Soret and thermal radiation on MHD fluid flow with viscous dissipation. The result showed that viscous dissipation enhanced temperature profiles while velocity decelerated with increase in thermal radiation.

Natural convection occurs when temperature changes in the fluid resulted to density variation, hence, the buoyancy forces act on the fluid elements. It has gained the attention of numerous researchers due to its applications in the real world which include: soil physics, oil reservoir, ground water flow, hydrology, nuclear physics, just to mention but a few. Nehad et al. (2019) examined free convection MHD flows over a vertical moving plate in permeable medium with thermal memory. Laplace transform method was used for the analysis and it was reported that the flow field was retarded due to the presence of the magnetic parameter. Kumar et al. (2017) presented effects of variable mass diffusion on natural convective fluid flow via an infinite vertical impulsively started plate. Iva et al. (2018) analysed the effect of heat generation in free convective MHD unsteady mass and heat transfer flow via an inclined surface. An explicit finite difference method was explored for the simplification of the differential equations involved. It was observed that velocity decelerated as a result of the influence of the magnetic parameter. Malleswari (2018) investigated natural convective Rivlin-Ericksen fluid along with the effects of double diffusion and transverse magnetic field on the flow past a vertical permeable plate. The result revealed that temperature decreased with increase in Prandtl number and heat absorption parameter.

Furthermore, Rangasamy and Murugesan (2018) discussed effects of Hall current and Soret on free convective unsteady fluid flow via a vertically infinite plate in the presence of variable permeability and velocity oscillatory suction. It was reported that temperature distributions are enhanced with increase in Eckert number. Fatunmbi and Odesola (2018) considered mass and heat transfer in natural convective MHD micropolar fluid flow on a stretching porous sheet in the presence of constant mass and heat flux. The study concluded that in comparison to Newtonian fluids, the shear stresses exhibited by micropolar fluid are reduced. Soret and radiation effects on free convective MHD micropolar fluid flow with mass transfer and uniform mass and heat fluxes 
was studied by Sengupta and Deb (2018). The result showed that linear velocity is enhanced with increase in dimensionless material parameter. Konwar (2018) examined the effects of chemical reaction and radiation on natural convective MHD fluid flow with mass and heat transfer in permeable medium. Marina et al. (2018) presented the thermal radiation effect on free convection in a permeable square cavity saturated with a temperature-dependent viscosity fluid.

However, the aforementioned studies ignored the combined effects of thermal radiation and viscous dissipation on the unsteady free Convective flow through an irregular channel. As a result of numerous applications of the thermal radiation on the fluid flow, the aim of the present work is to examine combined effects of thermal radiation and viscous dissipation on unsteady natural convective flow of an electrically conducting fluid through an irregular vertical channel in the presence of magnetic field. The resulting, coupled dimensionless non-linear ordinary differential equations (ODE) governing the fluid flow are perturbed and solved by employing ADM with Mathematica 11.0 software package, because of its flexibility in solving both linear and nonlinear ODE.

\section{Mathematical analysis}

A free convective, laminar, unsteady incompressible electrically conducting fluid flow past a wavy vertical channel in the presence of thermal radiation and viscous dissipation is considered. The $x$-axis which is vertically upward the channel is considered to be infinite. The irregular and flat wall is taken to be $\eta^{*}=\varepsilon^{*} \cos \omega x$ and $\eta^{*}=\lambda$, respectively, with the distance $\lambda$ apart. The uniform magnetic field $B_{o}$ is normal to $\eta$-axis, while $\eta$-axis is perpendicular to $x$-axis. The wavelength of the rough or irregular wall is $\omega=\frac{2 \pi}{k}$, where $\mathrm{k}$ is the coefficient of the wave.

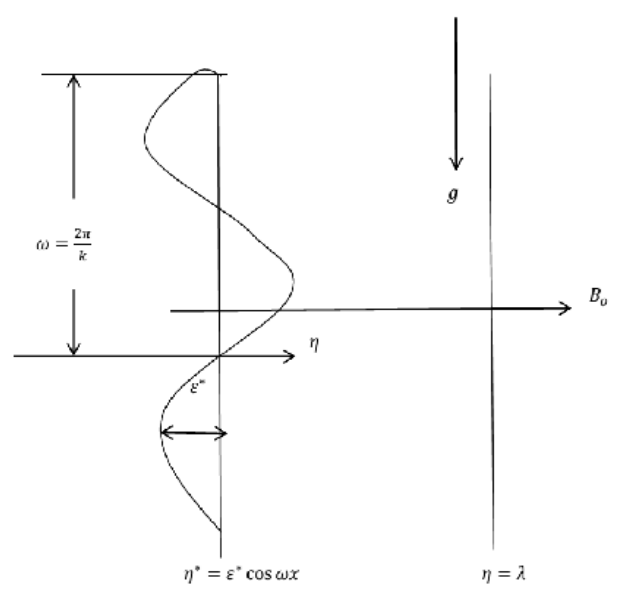

Figure 1. Physical configuration of the problem

With reference to Boussinesq approximation, the governing equations in dimensional forms are:

$$
\begin{gathered}
\frac{\partial \xi^{*}}{\partial t^{*}}=v \frac{\partial^{2} \xi^{*}}{\partial \eta^{* 2}}+g \beta_{\phi}\left(\phi^{*}-\phi_{\lambda}^{*}\right)-\frac{\sigma B_{o}^{2} \xi^{*}}{\rho} \\
\frac{\partial \phi^{*}}{\partial t^{*}}=\alpha \frac{\partial^{2} \phi^{*}}{\partial \eta^{* 2}}+\frac{\nu}{C_{P}}\left(\frac{\partial \xi^{*}}{\partial \eta^{*}}\right)^{2}-\frac{Q_{o}}{\rho C_{P}}\left(\phi^{*}-\phi_{\lambda}^{*}\right)-\frac{1}{\rho C_{p}} \frac{\partial q_{r}}{\partial \eta^{*}}
\end{gathered}
$$


with the following associated boundary conditions:

$$
\left.\begin{array}{l}
\xi^{*}=w_{o}\left(1+\varepsilon e^{n^{*} t^{*}}\right), \phi^{*}=\phi_{o}^{*}+\varepsilon\left(\phi_{o}^{*}-\phi_{\lambda}^{*}\right) e^{n^{*} t^{*}} \text { at } \eta^{*}=\varepsilon^{*} \cos \omega x, \\
\xi^{*}=0, \phi^{*}=\phi_{\lambda}^{*} \text { at } \eta^{*}=\lambda
\end{array}\right\}
$$

The radiative heat flux $q_{r}$ according to Rosseland approximation (Zigta and Koya 2017), is given as

$$
q_{r}=-\frac{4 \sigma^{*} \partial \phi^{* 4}}{3 k^{\prime} \partial \eta^{*}}
$$

The temperature difference is taken to be sufficiently small within the flow, hence using Taylor series expansion to expand $\phi^{4}$ and ignoring higher order terms leads to

$$
\phi^{* 4} \cong 4 \phi_{\lambda}^{* 3} \phi^{*}-3 \phi_{\lambda}^{* 4}
$$
below:

The non-dimensional governing equation is obtained using the non-dimensional variables

$$
\left.\begin{array}{l}
\eta=\frac{w_{o} \eta^{*}}{v}, G_{\phi}=\frac{v g \beta_{\phi}\left(\phi_{o}^{*}-\phi_{\lambda}^{*}\right)}{w_{o}^{3}}, P_{r}=\frac{w_{o} \rho C_{P}}{k}=\frac{v}{\alpha}, \psi=\frac{Q_{o} v}{w_{o}^{2} \rho C_{p}}, \xi=\frac{\xi^{*}}{w_{o}} \\
\varepsilon=\frac{\varepsilon^{*}}{\lambda}, t=\frac{t^{*} w_{o}^{2}}{v}, \phi=\frac{\phi^{*}-\phi_{\lambda}^{*}}{\phi_{o}^{*}-\phi_{\lambda}^{*}}, M=\frac{\sigma B_{o}^{2} v}{\rho w_{o}^{2}}, N=\frac{k^{\prime} k}{4 \sigma T_{\lambda}^{* 3}}, \mathrm{E}=\frac{w_{o}^{2}}{C_{P}\left(\phi_{o}^{*}-\phi_{\lambda}^{*}\right)}
\end{array}\right\}
$$

From eqs. (4) and (6), eqs. (1) and (2) become:

$$
\begin{gathered}
\frac{\partial \xi}{\partial t}=\frac{\partial^{2} \xi}{\partial \eta^{2}}+G_{\phi} \phi-M \xi \\
\frac{\partial \phi}{\partial t}=\frac{1}{P r} \frac{\partial^{2} \phi}{\partial \eta^{2}}+\mathrm{E}\left(\frac{\partial \xi}{\partial \eta}\right)^{2}-\psi \phi+\frac{4}{3 R P_{r}} \frac{\partial^{2} \phi}{\partial \eta^{2}}
\end{gathered}
$$

The boundary conditions associated to the flow are:

$$
\left.\begin{array}{l}
\xi=1+\varepsilon e^{n t}, \phi=1+\varepsilon e^{n t} \text { at } \eta=\varepsilon \cos \omega x, \\
\xi=0, \phi=0 \text { at } \eta=1
\end{array}\right\}
$$

\section{Method of Solution}

Regular Perturbation technique is applied to eqs. (7) and (8) resulting in coupled, non-linear ordinary differential equtions (ODE). The solutions to the ODE is obtained through Adomian Decomposition method (ADM), since ADM is applicable to both linear and non-linear differential equations (Chen and Lu 2004). Velocity and temperature assumed solutions (Raju et al. 2018), with reference to the boundary conditions, are expressed as

$$
\begin{gathered}
\xi(\eta, t)=\xi_{o}(\eta)+\varepsilon e^{n t} \xi_{1}(\eta)+0\left(\xi^{2}\right) \\
\phi(\eta, t)=\phi_{o}(\eta)+\varepsilon e^{n t} \phi_{1}(\eta)+0\left(\phi^{2}\right)
\end{gathered}
$$

Equations (10) and (11), reduced eqs. (7) and (8) to

$$
\begin{gathered}
\xi_{o}^{\prime \prime}-M \xi_{o}+G_{\phi} \phi_{o}=0 \\
\xi_{1}^{\prime \prime}-(M+n) \xi_{1}+G_{\phi} \phi_{1}=0 \\
Q \phi_{o}^{\prime \prime}-\psi \phi_{o}+E\left(\xi_{o}^{\prime}\right)^{2}=0 \\
Q \phi_{1}^{\prime \prime}-(\psi+n) \phi_{1}+2 \mathrm{E} \xi_{o}^{\prime} \xi_{1}^{\prime}=0
\end{gathered}
$$

where 


$$
Q=\frac{1}{P r}+\frac{4}{3 \mathrm{~N} P r}
$$

The boundary conditions, similarly, become

$$
\left.\begin{array}{l}
\xi_{o}=1, \xi_{1}=1, \phi_{o}=1, \phi_{1}=1 \text { at } \eta=\varepsilon \cos \omega x, \\
\xi_{o}=0, \xi_{1}=0, \phi_{o}=0, \phi_{1}=0 \text { at } \eta=1
\end{array}\right\}
$$

Following Gbadeyan et al. (2017), $\varepsilon$ is taken to be zero which is the limit for the smooth flat wall. Therefore, $\eta=\varepsilon \cos \omega x=0$. The initial approximation and recurrence relation for eqs. (12)(15) with the boundary conditions (16) is given as follows:

$$
\begin{gathered}
\left(\xi_{0}\right)_{0}=1+b_{1} \eta \\
\left(\xi_{1}\right)_{0}=1+b_{2} \eta \\
\left(\phi_{0}\right)_{0}=1+b_{3} \eta \\
\left(\phi_{1}\right)_{0}=1+b_{4} \eta \\
\left(\xi_{0}\right)_{r+1}=\int_{0}^{\eta} \int_{0}^{s}\left(M\left(\xi_{0}\right)_{r}-G_{\phi}\left(\phi_{0}\right)_{r}\right) d \eta d s ; \\
\left(\xi_{1}\right)_{r+1}=\int_{0}^{\eta} \int_{0}^{s}\left((M+n)\left(\xi_{1}\right)_{r}-G_{\phi}\left(\phi_{1}\right)_{r}\right) d \eta d s ; \\
\left(\phi_{0}\right)_{r+1}=\int_{0}^{\eta} \int_{0}^{s} \frac{1}{Q}\left(\psi\left(\phi_{0}\right)_{r}-\mathrm{E}\left(\left(\xi_{0}^{\prime}\right)_{r}\right)^{2}\right) d \eta d s ; \\
\left(\phi_{1}\right)_{r+1}=\int_{0}^{\eta} \int_{0}^{s} \frac{1}{Q}\left((\psi+n)\left(\phi_{1}\right)_{r}-2 \mathrm{E}\left(\xi_{0}^{\prime}\right)_{r}\left(\xi_{1}^{\prime}\right)_{r}\right) d \eta d s
\end{gathered}
$$

where $r=0,1,2, \ldots$

The solution to eqs. (7) and (8) is obtained in series form. These series converge at $r=5$ and can be expressed as:

$$
\begin{gathered}
\xi(\eta, t)=\sum_{r=0}^{5}\left(\xi_{0}\right)_{r}+\varepsilon e^{n t} \sum_{r=0}^{5}\left(\xi_{1}\right)_{r} \\
\phi(\eta, t)=\sum_{r=0}^{5}\left(\phi_{0}\right)_{r}+\varepsilon e^{n t} \sum_{r=0}^{5}\left(\phi_{1}\right)_{r}
\end{gathered}
$$

\section{Results and Discussion}

In order to examine the behaviour of each involved parameter in the flow on the physical problem, the computational analysis is carried out on Partial Differential Equations (PDE) (7) and (8) with the boundary conditions (9) using ADM along with MATHEMATICA 11.0 software. The effects of various parameters for velocity and temperature profiles are presented in graphs, while the Skin friction and Nusselt number are presented in the table. The following parameters are considered: Prandtl number $P_{r}$, magnetic parameter $M$, dimensionless heat absorption coefficient $\psi$, radiation parameter $N$, dissipation parameter $\mathrm{E}$ and Grashof number for heat transfer $G_{\phi}$. The following are the default values used for the computations (Ajibade and Kabir 2019) and (Agunbiade and Dada 2019): $M=0.3, P_{r}=0.71, N=2.0, \psi=3, G_{\phi}=7.0, \mathrm{E}=0.5, \varepsilon=0.2$, $n=0.5$ and $t=1$. 


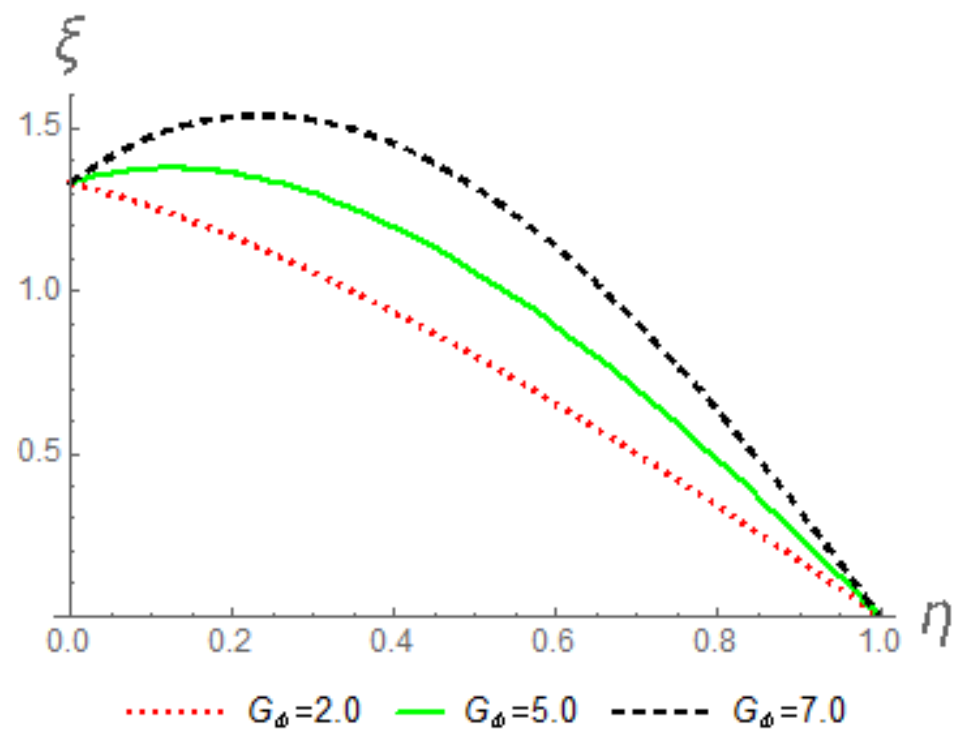

Figure 2. Effects of $G_{\phi}$ on $\xi$

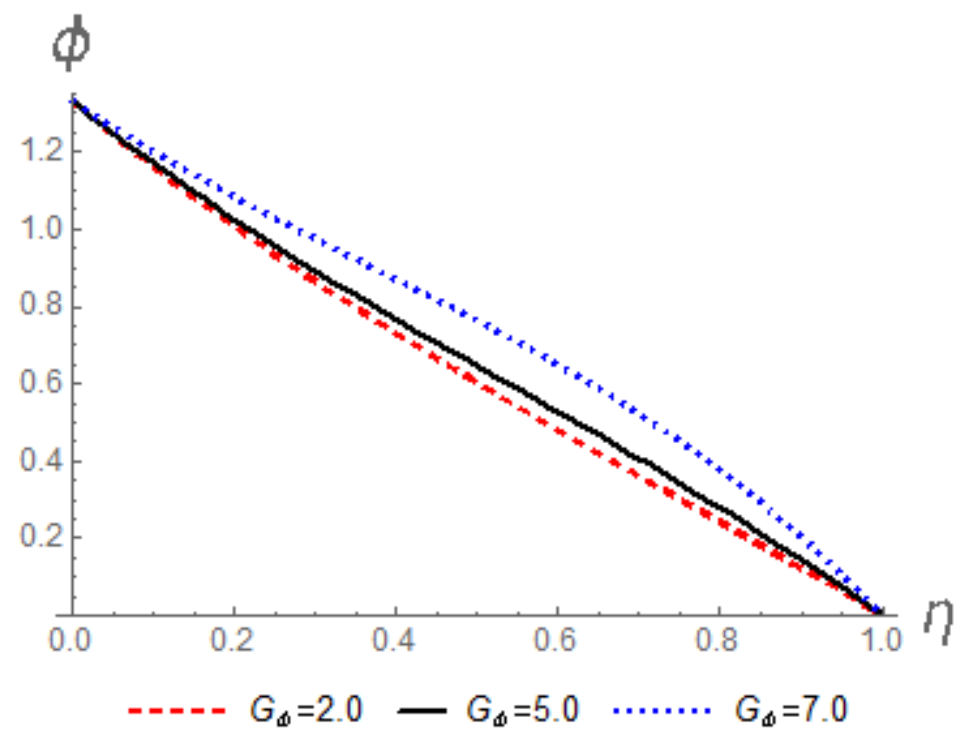

Figure 3. Effect of $G_{\phi}$ on $\phi$

Figures 2 and 3 depict the effect of Grashof number for heat transfer $G_{\phi}$ on velocity and temperature respectively. Velocity and temperature distributions accelerated throughout the flow region with increase in $G_{\phi}$. In the Physical, buoyancy force is enhanced for higher values of Grashof number, hence hydrodynamics increases within the channel. It is noticed that the cooling of the surface corresponds to positive values of $G_{\phi}$. 


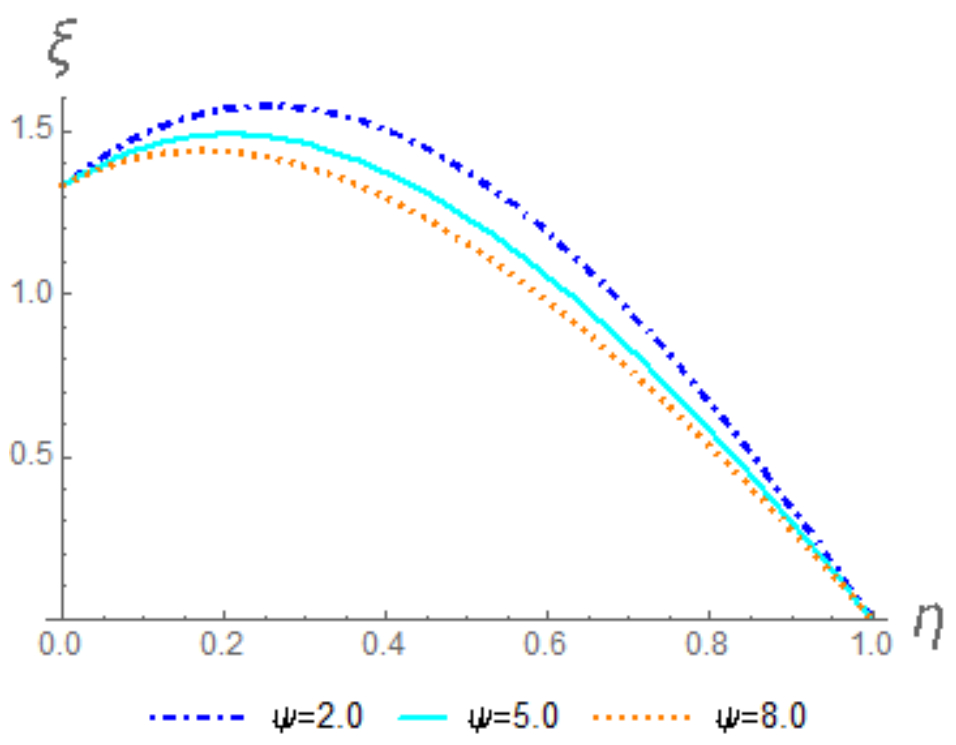

Figure 4. Effects of $\psi$ on $\xi$

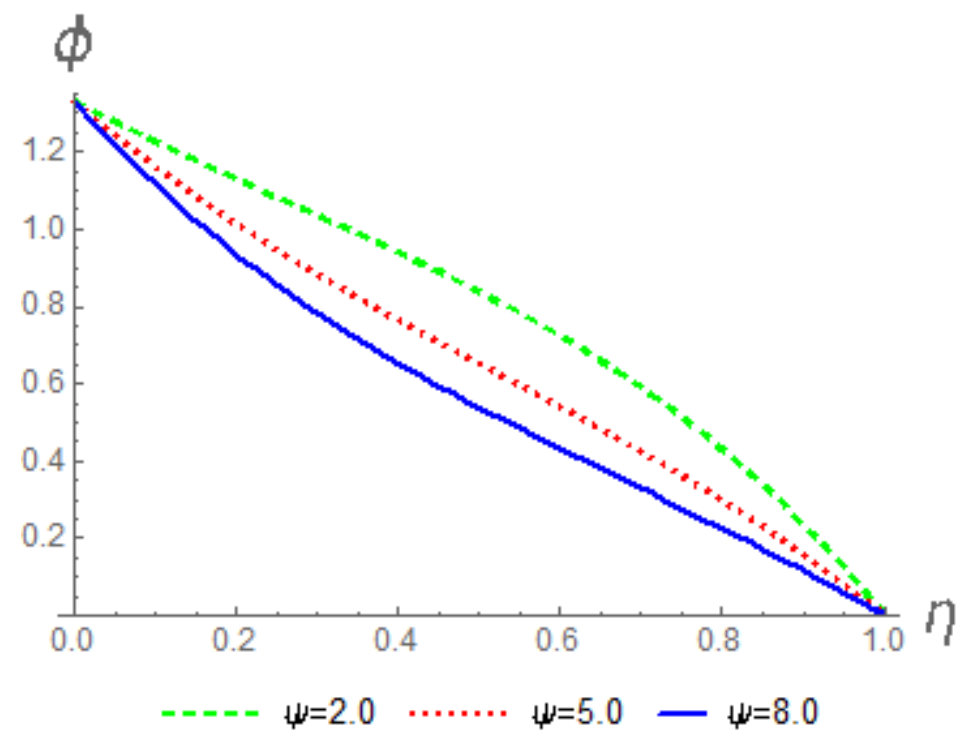

Figure 5. Effect of $\psi$ on $\phi$

The variation of heat absorption coefficient $\psi$ on velocity and temperature profiles is showed in Figs. 4 and 5. From these figures, it is noticed that the temperature and velocity profiles experienced decline with a rise in $\psi$ parameter. Physically, $\psi$ has the tendency of retarding the fluid temperature. Due to absorption of heat, the temperature profiles is decreased by the buoyancy force effects, hence, the flow rate is consequently slowdown. 


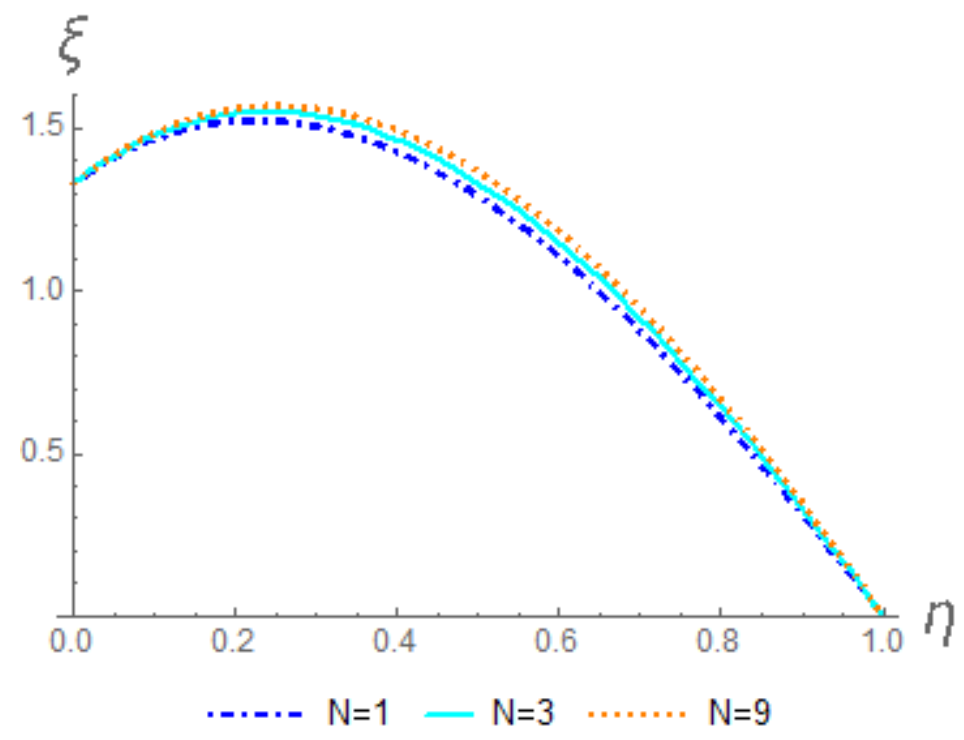

Figure 6. Effects of $N$ on $\xi$

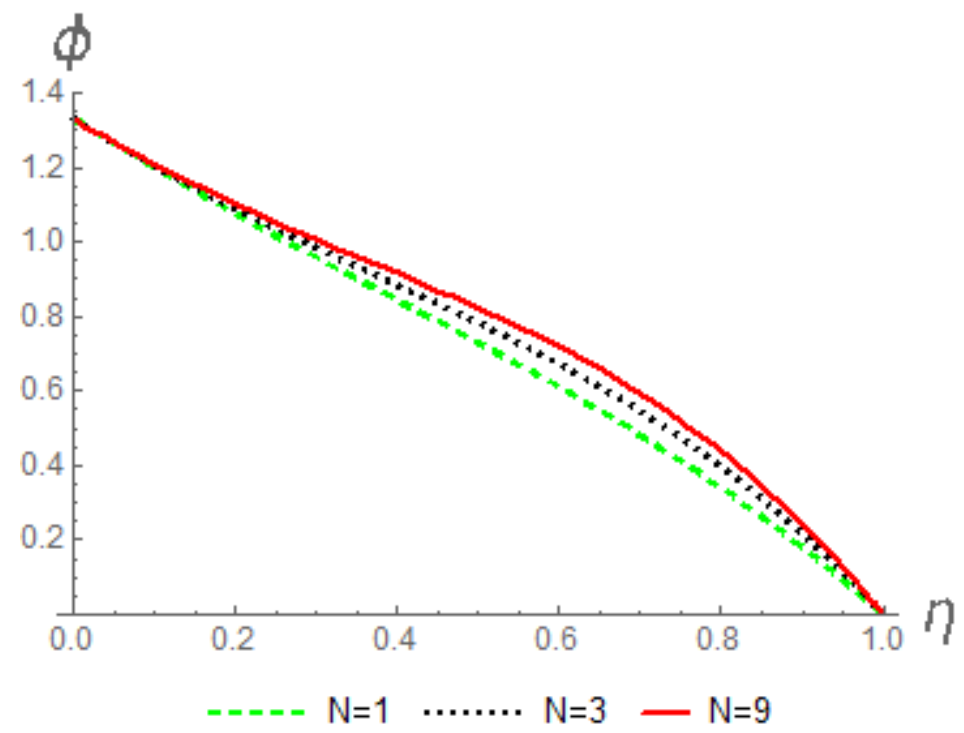

Figure 7. Effect of $N$ on $\phi$

The effect of the radiation parameter $N$ is revealed in Figs. 6 and 7. It is obvious from these figures that a hike in the values of $N$ led to a rise in velocity and temperature profiles. This fact may be attributed to diminishing effect of Roseland radiative absorption parameter, which corresponds to divergence of the heat flux, hence increasing the radiative heat transfer rate of the fluid that resulted in a rise in the fluid temperature. 


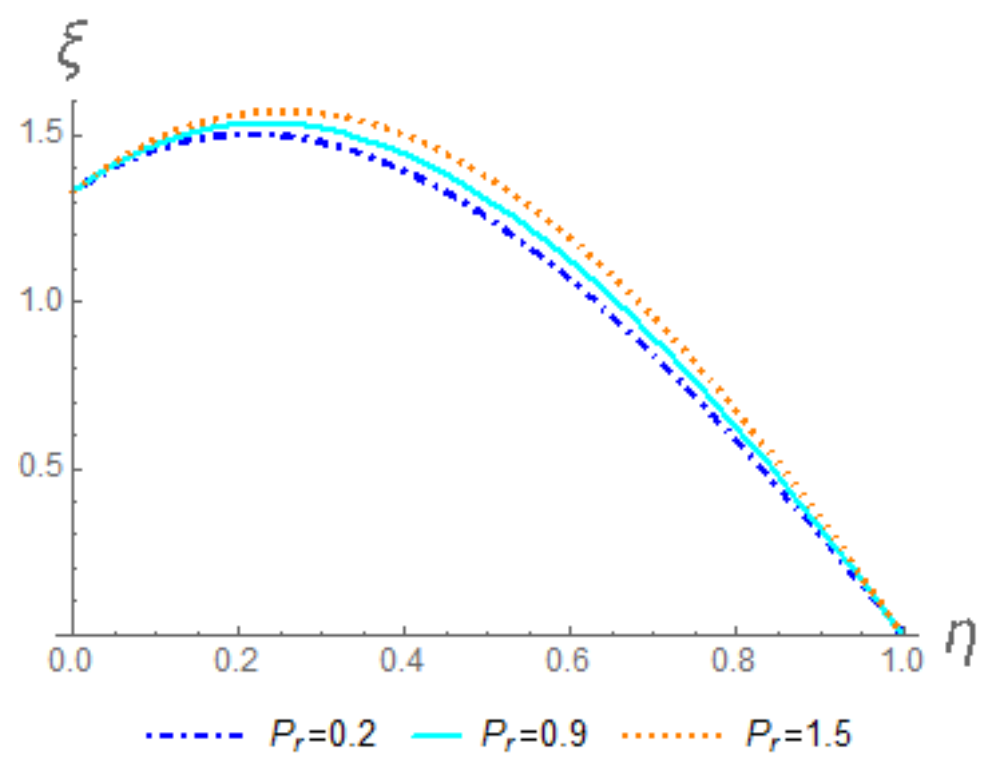

Figure 8. Effect of $P_{r}$ on $\xi$

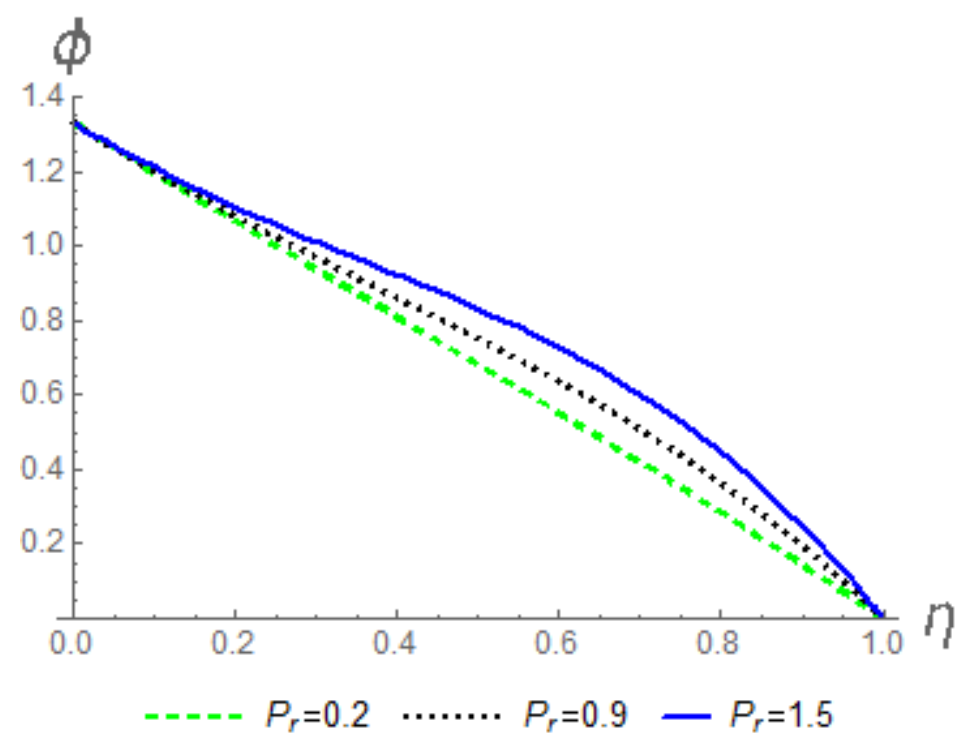

Figure 9. Effect of $P_{r}$ on $\phi$

Figures 8 and 9 show the variation of Prandtl number $P_{r}$ on the velocity and temperature profiles. Both the velocity and the temperature profiles are enhanced with increase in Prandtl number $P_{r}$. In practice, thermal conductivity of the fluid decelerated due to a rise in Prandtl number. Hence, the rate of diffusion of the heat becomes more rapid at the heated surface for higher values of Prandtl number. 


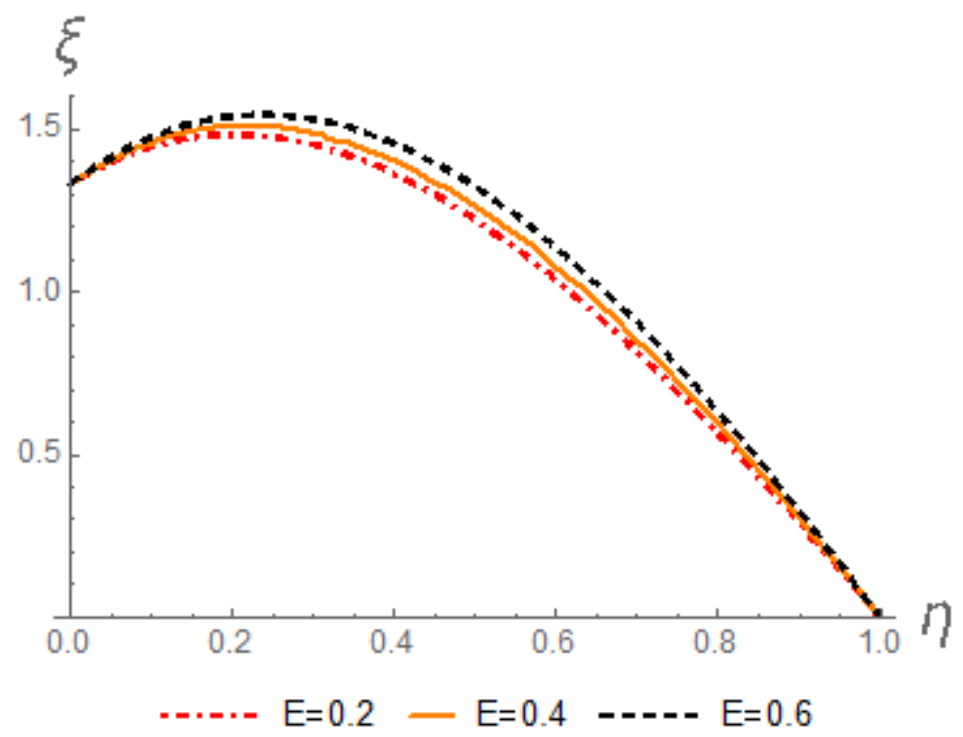

Figure 10. Effects of $E$ on $\xi$

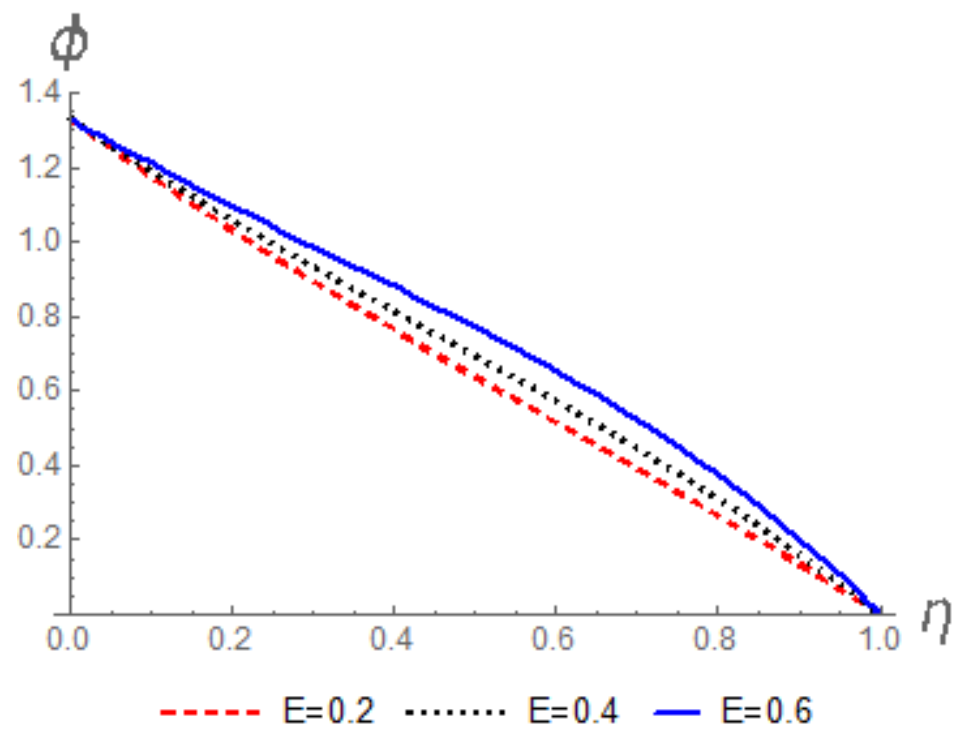

Figure 11. Effect of $E$ on $\phi$

The variation of the viscous dissipation is exhibited in Figs. 10 and 11 for the velocity and temperature profiles. The kinetic energy and enthalpy relationship is displayed through Dissipation. It is observed that both the velocity and the temperature profiles speedup with increase in E. Energy increases as a result of the effect of dissipation on the fluid, consequently, increases the fluid buoyancy force and temperature. Therefore, it is shown from the Figures that the temperature and the velocity are enhanced as the values of $E$ become higher. 


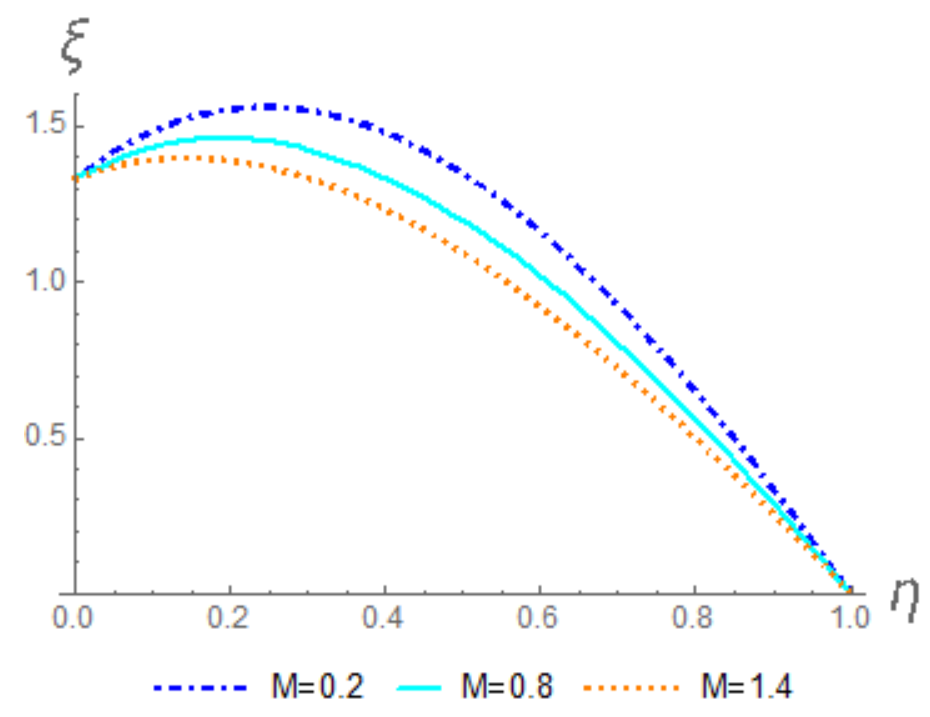

Figure 12. Effects of $M$ on $\xi$

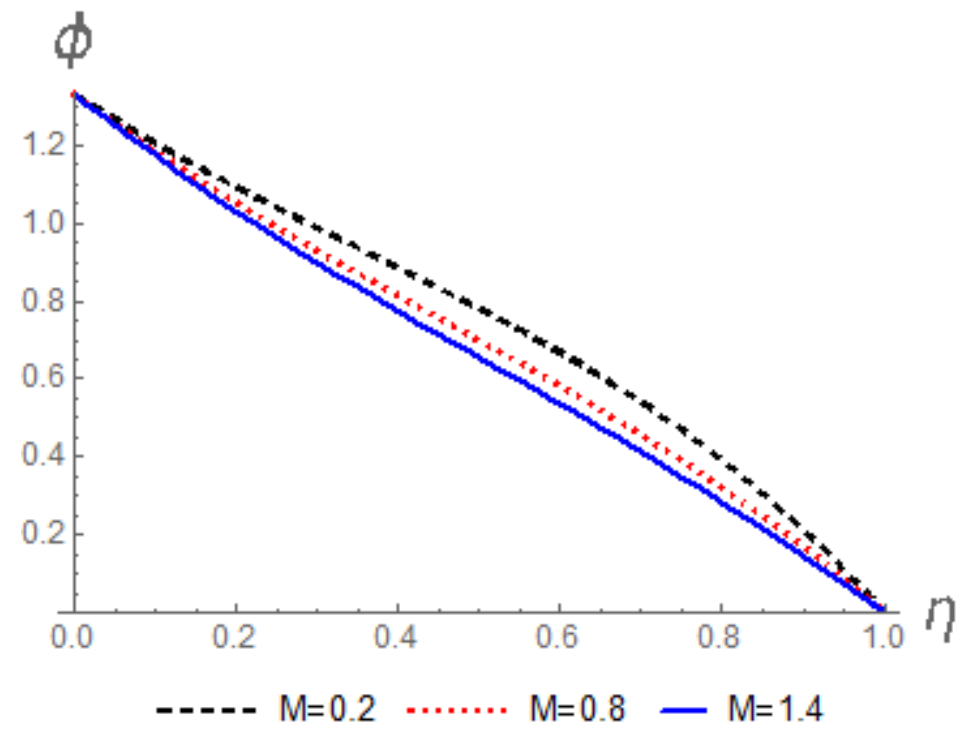

Figure 13. Effect of $M$ on $\phi$

Influence of the magnetic parameter $M$ on velocity and temperature profiles is displayed in Figs. 12 and 13. Increase in $M$ slowed down velocity profiles which is shown in Fig. 12. The application of the magnetic field in electrically conducting fluid normally produces a drag force often referred to as Lorentz force. This drag force opposes the fluid motion, therefore, the velocity of the fluid is retarded as a result. Similarly, temperature profiles experience a decline with a hike in magnetic parameter. 


\begin{tabular}{|c|c|c|c|c|c|c|c|}
\hline$N$ & $\mathrm{E}$ & $P_{r}$ & $\psi$ & $G_{\phi}$ & $M$ & $S_{f}$ & $N u$ \\
\hline 1 & 0.5 & 0.71 & 3.0 & 7.0 & 0.3 & 1.779700 & -1.351200 \\
\hline 3 & 0.5 & 0.71 & 3.0 & 7.0 & 0.3 & 1.887350 & -1.323490 \\
\hline 9 & 0.5 & 0.71 & 3.0 & 7.0 & 0.3 & 1.971490 & -1.288950 \\
\hline 1.0 & 0.2 & 0.71 & 3.0 & 7.0 & 0.3 & 1.579600 & -1.597350 \\
\hline 1.0 & 0.4 & 0.71 & 3.0 & 7.0 & 0.3 & 1.702260 & -1.446050 \\
\hline 1.0 & 0.6 & 0.71 & 3.0 & 7.0 & 0.3 & 1.872690 & -1.237710 \\
\hline 1.0 & 0.5 & 0.2 & 3.0 & 7.0 & 0.3 & 1.685180 & -1.344200 \\
\hline 1.0 & 0.5 & 0.9 & 3.0 & 7.0 & 0.3 & 1.823710 & -1.342870 \\
\hline 1.0 & 0.5 & 1.5 & 3.0 & 7.0 & 0.3 & 1.988420 & -1.281140 \\
\hline 1.0 & 0.5 & 0.71 & 2.0 & 7.0 & 0.3 & 2.020870 & -1.047160 \\
\hline 1.0 & 0.5 & 0.71 & 5.0 & 7.0 & 0.3 & 1.588650 & -1.804250 \\
\hline 1.0 & 0.5 & 0.71 & 8.0 & 7.0 & 0.3 & 1.321010 & -2.351550 \\
\hline 1.0 & 0.5 & 0.71 & 3.0 & 2.0 & 0.3 & -0.618170 & -1.758190 \\
\hline 1.0 & 0.5 & 0.71 & 3.0 & 5.0 & 0.3 & 0.716803 & -1.667320 \\
\hline 1.0 & 0.5 & 0.71 & 3.0 & 7.0 & 0.3 & 1.847120 & -1.336600 \\
\hline 1.0 & 0.5 & 0.71 & 3.0 & 7.0 & 0.2 & 1.954950 & -1.286830 \\
\hline 1.0 & 0.5 & 0.71 & 3.0 & 7.0 & 0.8 & 1.390840 & -1.514280 \\
\hline 1.0 & 0.5 & 0.71 & 3.0 & 7.0 & 1.4 & 0.956761 & -1.634700 \\
\hline
\end{tabular}

Table 1. Values of Skin-friction and Nusselt number for different parameters.

\begin{tabular}{|c|cc|cc|cr|}
\hline & \multicolumn{2}{|c|}{ Jha and Ajibade } & \multicolumn{2}{c|}{ Ajibade and Kabir } & \multicolumn{2}{c|}{ Present work } \\
\hline$\psi$ & \multicolumn{2}{|c|}{ Temperature } & Velocity & Temperature & Velocity & \multicolumn{2}{c|}{$\begin{array}{c}\text { Temperature } \\
\text { Velocity }\end{array}$} \\
\hline-1.0 & 0.569747 & 1.067976 & 0.568331 & 1.064149 & 0.569747 & 1.057980 \\
-0.5 & 0.532965 & 1.029436 & 0.535019 & 1.029058 & 0.532965 & 1.027440 \\
0.5 & 0.470299 & 0.975218 & 0.470377 & 0.975282 & 0.470299 & 0.975218 \\
1.0 & 0.443409 & 0.952725 & 0.443485 & 0.953983 & 0.443409 & 0.952724 \\
\hline
\end{tabular}

Table 2. Comparison of the present work with the work of Jha and Ajibade (2010) and Ajibade and Kabir (2019) $\left(P_{r}=0.71, G_{\phi}=8.0\right.$ and $\left.y=\eta=0.5\right)$

Table 1 presents the variation of various fluid parameters $\left(N, \mathrm{E}, P_{r}, \psi, M\right.$, and $\left.G_{\phi}\right)$ on $S_{f}$ and $N u$. For $\eta=0$, it is depicted that a rise in $G_{\phi}, P_{r}$, and E enhanced both the skin friction and the heat transfer coefficient. While, $S_{f}$ and $N u$ experience a retardation with increase in $\psi$ and M.

Table 2 displayed the comparison of this present study with the work of Jha and Ajibade (2010) and Ajibade and Kabir (2019). By setting $M=\mathrm{E}=n=\varepsilon=t=0$, the present work is in agreement with the work of Jha and Ajibade (2010) and Ajibade and Kabir (2019) is shown in Table 2. 


\section{Conclusion}

In this paper, combined effects of thermal radiation and viscous dissipation on unsteady free Convective flow of an incompressible and electrically conducting fluid through an irregular channel are studied in the presence of the magnetic field. The dimensionless PDE governing the flow is reduced to ODE with the help of perturbation technique. The resultant coupled, non-linear ODE was solved applying ADM using Mathematica 11.0 software. The results are presented graphically showing the effects of different parameters on fluid. In addition, for scientific purposes, Skin friction and Nusselt number are illustrated in tabular form. It is discovered that velocity, temperature, Skin friction and rate of heat transfer are enhanced with increase in $N, \mathrm{E}$, $P_{r}$ and $G_{\phi}$. However, they are retarded with a rise in $\psi$ and $M$. The results show that combined effects of thermal radiation and viscous dissipation have significant impacts on the fluid flow, hence it can be used in controlling the cooling rate of machines operating at high temperature.

\section{Nomenclature}

\begin{tabular}{|c|c|}
\hline$C_{p}$ & specific heat (constant pressure) $\left(J k g^{-1} K^{-1}\right)$ \\
\hline$G_{\phi}$ & thermal Grashof number \\
\hline$B_{0}$ & magnetic field induction (tesla) \\
\hline$P_{r}$ & Prandtl parameter \\
\hline$w_{o}$ & the wavy wall velocity $\left(m s^{-1}\right)$ \\
\hline$M$ & magnetic parameter \\
\hline$g$ & gravitational acceleration $\left(m / s^{2}\right)$ \\
\hline$N$ & thermal radiation \\
\hline $\mathrm{E}$ & Dissipation parameter \\
\hline$Q_{o}$ & heat generation absorption/ coefficient $\left(\mathrm{Kgm}^{-1} \mathrm{~s}^{-3} \mathrm{~K}^{-1}\right)$ \\
\hline$t$ & time \\
\hline$n$ & index of exponential \\
\hline \multicolumn{2}{|c|}{ Greek Symbols } \\
\hline$\phi_{o}$ & left wall temperature $(\mathrm{K})$ \\
\hline$\phi_{\lambda}^{*}$ & dimensional temperature at the channel wall $(\mathrm{K})$ \\
\hline$\mu$ & viscosity coefficient \\
\hline$\xi^{*}$ & dimensional velocity components in the $\eta^{*}$ directions $\left(m s^{-1}\right)$ \\
\hline$\lambda$ & distance between the plates $(\mathrm{m})$ \\
\hline$\rho$ & the density fluid $\left(\mathrm{kg} \mathrm{m}^{-3}\right)$ \\
\hline$\phi^{*}$ & temperature in dimensional form $(\mathrm{K})$ \\
\hline$v^{*}$ & the kinematic viscosity $\left(m^{2} s^{-1}\right)$ \\
\hline$\beta_{T}$ & the coefficient of the thermal expansion $\left(K^{-1}\right)$ \\
\hline$\psi$ & heat absorption parameter in non-dimensional form \\
\hline$\alpha$ & thermal diffusivity $\left(s^{2} / m\right)$ \\
\hline$\eta^{*}$ & the plate perpendicular dimensional distance $(\mathrm{m})$ \\
\hline$\sigma$ & electrical conductivity \\
\hline$\varepsilon$ & Scalar Constant \\
\hline
\end{tabular}




\section{References}

Agunbiade SA and Dada MS (2019). Effects of viscous dissipation on convective rotatory chemically reacting Rivlin-Ericksen flow past a porous vertical plate. Journal of Taibah University for Science, 13 (1): 402-413. DOI: 10.1080/16583655.2019.1582149

Ajibade AO and Kabir TM (2019). Viscous dissipation effect on steady natural convection couette flow of heat generating fluid in a vertical channel. Journal of Advances in Mathematics and Computer Science, 30 (1): 1-16. DOI:10.9734/JAMCS/2019/45020

Chen W and Lu Z (2004). An algorithm for Adomian decomposition method. Applied Mathematics and Computation, 159: 221-235.

Dada, M. S., Agunbiade, S. A., (2016). Radiation and chemical reaction effects on convective Rivlin-Ericksen flow past a porous vertical plate. Ife Journal of Science. 18 (3), 655-667.

Emeka A (2019). Effect of viscous dissipation and Soret on MHD flow with thermal radiation. International Journal of Applied Science and Mathematical theory, 5 (1): 57-65.

Fatunmbi EO and Odesola AS (2018). MHD free convective heat and mass transfer of a micropolar fluid flow over a stretching permeable sheet with constant heat and mass flux. Asian Research Journal of Mathematics, 9 (3): 1-15. DOI:10.9734/ARJOM/2018/40823

Gbadeyan JA, Oyekunle TL and Abubakar JU (2017). Thermal radiation and chemical reaction effects on free convective heat and mass transfer flow through an irregular channel. FUW Trends in Science and Technology Journal (FTSTJ) 2 (1B): 462-477.

Iva LM, Sawpna MA, Chanda RK and Mondal RN (2018). Unsteady MHD free convective heat and mass transfer flow past an inclined surface with heat generation. The international Journal of Engineering and Science, 7 (10): 68-76. DOI: 10.9790/1813-0710016876

Jha BK and Ajibade AO (2010). Unsteady free convective Couette flow of heat generating/absorbing fluid. International Journal of Energy and Technology, 2 (12) : 1-9.

Jhankal AK, Jat RN and Kumar D (2017). Magnetohydrodynamics (MHD) forced convective flow and heat transfer over a porous plate in a Darcy-forchheimer porous medium in presence of radiation. International Journal Cur. Res. Rev., 9 (11): 16-23. DOI:http://dx.doi.org/10.7324/IJCRR.2017.9114

Konwar H 2018. Radiation and chemical reaction effects on MHD free convection flow, heat and mass transfer past a permeable plate in porous medium. International Journal of research in Advent Technology, 16 (8): 2227-2231.

Kumar BR, Jayakar R and Kumar AGV 2017. Variable mass diffusion effects on free convection flow past an impulsively started infinite vertical plate. IOP Conf. Series:Materials Science Engineering, 263:062002. doi:10.1088/1757-899X/263/6/062002

Kumar DR and Reddy KJ (2018). Viscous dissipation and radiation effects on MHD convective flow past a vertical porous plate with injection. International Journal of Engineering Science Invention(IJESI), 7 (1): 26-32.

Malleswari D ( 2018). Influence of viscoelastic Rivlin-Ericksen fluid on free convective flow past a vertical plate filled in porous medium in the presence of transverse magnetic field and Double diffusion effects. Journal of Applied Physics, 10 (3): 45-62. DOI:10.9790/48611003024562

Marina SA, Mikhail AS and Jawali CU (2018). Effect of thermal radiation on Natural convection in a square porous cavity filled with a fluid of temperature-dependent viscosity. Thermal Science, 22 (1B): 391-399. DOI:10.2298/TSC1150722164A

Mostafa FB, Samad MA and Hossain MR (2017). Combined effect of viscous dissipation and radiation on unsteady free convective Non-Newtonian fluid along a continuously moving vertically stretched surface with No-Slip phenomena. American Journal of computational and Applied Mathematics, 7 (3): 71-79. DOI: 10.5923/j.ajcam.20170703.02 
Nagasasikala M and Lavanya B (2018). Effects of dissipation and radiation on heat transfer flow of a convective rotating cuo-water Nano-fluid in a vertical channel. Journal of Advanced Research in fluid Mechanics Thermal Sciences, 50 (2): 108-117.

Nehad AS, Najma A,Thanaa E and Mohammed MR (2019). Magnetohydrodynamic free convection flows with thermal memory over a moving vertical plate in porous medium. Journal of Applied and Computational Mechanics, 5 (1): 150-161. DOI:10.22055/JACM.2018.25682.1285

Raju VN, Hemalatha K and Babu VS (2018). Radiation effects on MHD convective heat and mass transfer flow past a semi-infinite vertical moving porous plate in the presence of chemical reaction. International Journal of engineering Sciences and Research Technology, 7 (8): 146-161. DOI:10.5281/zenodo. 1336713

Rao ME (2018). The effect of thermal radiation and chemical reaction on MHD flow of a Casson fluid over and exponentially inclined stretching surface. Journal of Physics:Conf. Series, 1000: 012158. doi:10.1088/1742-6596/1000/1/012158

Sengupta S and Deb R (2018). MHD free convection mass transfer flow of radiative micropolar fluid with Soret effect and uniform heat and mass fluxes. American Journal of heat and mass transfer, 5 (1): 49-75. doi:10.7726/ajhmt.2018.1004

Sivaiah G and Reddy K (2017). Unsteady MHD heat and mass transfer flow of a radiating fluid past an accelerated inclined porous plate with Hall current. International Journal of Research-Granthaalayah, 5 (7): 42-59. DOI: 10.5281/zenodo. 826850

Suguna HG and Bhuvana VR (2019). Effect of Hall current on mixed convection squeezing three dimensional flow in a rotating channel with thermal radiation and heat sources. International Journal of Scientific and innovative mathematical Research (IJSIMR), 7 (3): 40-52. DOI:http://dx.doi.org/10.20431/2347-3142.0703005

Rangasamy P and Murugesan N (2018). Soret and hall effect on unsteady free convective flow past an infinite vertical plate with oscillatory suction velocity and variable permeability. International Journal of heat and Technology, 36 (3): 808-816. DOI: https://doi.org/10.18280/ijht.360035

Zigta B and Koya PR (2017). The effect of MHD on free convection with periodic temperature and concentration in the presence of thermal radiation and chemical reaction. International Journal of Applied Mechanics and Engineering, 22 (4): 1059-1073. DOI: 10.1515/ijame2017-0068 